\title{
Gesellschaftliche Umverteilung, ein moralisches Muss? Verwundbarkeit und soziale Sicherung im Zeichen gesellschaftlichen Umbruchs - Beobachtungen aus Tansania in akteursorientierter Interpretation
}

\section{Sabine Tröger, Bayreuth}

\section{Soziale Sicherheit - ein Schutzfaktor bei Nahrungsdefiziten}

Mit der Beobachtung, dass Menschen mit vergleichbar defizitärer materieller Ressourcenlage in ihrer Nahrungssicherheit deutlich unterschiedlich verwundbar sind, rückt der Aspekt der «Sozialen Sicherheit» mit in den Vordergrund der Erforschung von Nahrungskrisen. Es stellt sich die Frage, in welcher Weise und unter welchen Bedingungen Mechanismen der gesellschaftlichen Umverteilung im Sinne einer Herabsetzung von Verwundbarkeit, die hier in Anlehnung an VOGEL (1998) als «characteristics of persons and groups in terms of their capacity to anticipate, cope with, resist, and recover from the impacts of environmental change» definiert wird, mit wirksam werden und von nahrungsunsicheren Menschen als «Sozialkapital» - «Social Capital» (DFID 1999) - zur Absicherung ihres Lebens zum Einsatz gebracht werden können.

Menschen in einer materiellen Notlage, aus der sie sich nicht mit eigenen Mitteln und Kräften befreien können, bedürfen der Hilfe und Unterstützung von außen. Eine Umverteilung zugunsten solcherart Bedürftiger ist für den Gebenden nicht in jedem Fall entbehrungsfrei, und so kann sich ein System des innergesellschaftlichen Ausgleichs mit dem Ziel einer Überlebenssicherung für alle nicht allein auf das Prinzip der Freiwilligkeit stützen. In Gesellschaften, deren Mitglieder nicht durch eine institutionelle Verankerung problemangemessener Hilfsstrukturen in einem Netz der Fürsorge aufgefangen werden, sind Mechanismen und Wirkungskräfte notwendig, die den Notleidenden ein Mindestma $\beta$ an Hilfsleistungen garantieren. In diesem Zusammenhang rückt in der wissenschaftlichen Diskussion der Aspekt der «Moral» und des «moralischen Handelns» in den Mittelpunkt des Interesses und wird aus der Perspektive zweier gegensätzlicher Grundpositionen betrachtet. Bereits die Frage, ob überhaupt normative Vorgaben geeignet und wirksam erscheinen, Bedürftige zu schützen, wird von manchen grundsätzlich bejaht (z.B. Scotr 1976: 6-7) und von anderen verneint (z.B. POPKIN 1979: 17 und 30). Eine zwischen diesen beiden Polen vermittelnde Position vertritt Platteau (1991: 155, vgl. auch ZedLITZ 1993, SchmidT 1992), wenn er auf die notwendige Kongruenz zwischen Regelvorgaben und normierten Handlungskodizes auf der einen und Hand- lungsmotiven auf der anderen Seite verweist, die aus der individuellen Interessenlage resultieren.

Eine Bereitschaft zur Umverteilung wird fernerhin in der Diskussion mit spezifischen Konditionen in Verbindung gebracht, auf deren Ausprägung die Subjekte nur begrenzt oder gar keinen Einfluss haben. So wird zum Beispiel eine insgesamt ausreichende ökonomische Basis als unabdingbar für gesellschaftliche Umverteilung bewertet (Platteau 1991: 157, ZedLITZ 1994: 254). Daneben finden auch soziale Aspekte wie die physische und psychische Nähe zwischen den Menschen einer Gemeinschaft und mit ihnen die Frage der sozialen Interdependenz eine besondere Beachtung in der wissenschaftlichen Auseinandersetzung (z.B. PARTSCH 1983: 100).

Solche Bedingungen des moralischen Handelns sind jedoch dem historischen Wandel unterworfen, der in der einschlägigen Literatur besonders mit dem Blick auf die Auswirkungen der Arbeitsmigration, der verschärften sozio-ökonomischen Differenzierung in Folge zunehmender Marktintegration und der Maßnahmen von einer Strukturanpassung und moderner Einflüsse, welche bisherige Werte und Normen des Lebens in Gemeinschaft in ihrer Relevanz infrage stellen, Beachtung erfährt. Ob diese historischen Prozesse zu einer Herabsetzung der Bereitschaft zur Umverteilung oder eher zur Stärkung eben dieser Bereitschaft und damit zu mehr sozialer Sicherheit führen, ist Gegenstand einer breiten wissenschaftlichen Diskussion, die bisher noch zu keinem befriedigend verallgemeinerbaren Ergebnis gelangt ist (vgl. BOSSERT 1988: 213, SCOTt 1976: 7, Popkin 1979: 30, SCHMidt 1992: 34, Platteau 1991: 163, Benda-Beckmann et al. 1988: 13). In der Zusammenschau der Argumente zeichnet sich jedoch eine in der Tendenz eher negative Einschätzung der Wirkung moderner Entwicklungen auf die Absicherung der Menschen durch ihre Gemeinschaft ab, also eine Interpretation, die eine Entwicklung vom Guten zum Schlechten feststellt und die in der Regel mit einer aus dem historischen Wandel resultierenden Verengung der Spielräume für solidarisches Handeln begründet wird (DE BRUIJN \& VAN DIJK 1994: 200, SCHMidt 1992: 33, ZedLITZ 1994: 242).

\section{Zur Dynamik von Krisenbewältigung}

Folgen wir KASPERSON (2001: 3), so dürfen wir uns 
allgemein ein Handeln zur Krisenbewältigung jedoch nicht als starres, festgefügtes Handlungsmuster vorstellen. Mit der Konfrontation mit sich historisch wandelnden Rahmenvorgaben geht vielmehr ein Prozess des sozialen Lernens einher - "social learning involved in continuing encounters with stress»- der die Krisenbewältigung dynamisiert. In der Konsequenz erfährt die Interpretation dessen, was mit «Handeln» in dem Zusammenhang einer existentiellen Bedrohung gemeint sein kann, eine Ausrichtung, die zu der Frage überleitet, welcher Freiheitsgrad und Grad der Selbstbestimmtheit einem Handeln in solchen Situationen innewohnt. Das heißt, wir schließen uns nicht der Annahme einer grundsätzlichen durch Rahmenbedingungen vorgegebenen Determination der in ihrer Nahrungssicherheit bedrohten Menschen an, sondern suchen nach den Bedingungen und Artikulationsformen eines Handelns, das sich im Verlauf eines Lernprozesses dem historischen Wandel eigenbestimmt anpasst.

In diesem Zusammenhang bieten theoretische Überlegungen von Giddens (1976, in deutscher Übersetzung 1997) eine Interpretationshilfe. Gemäß der dort vertretenen Argumente werden «Handeln» und «Struktur» über den Prozess der «Strukturation» zueinander in Beziehung gesetzt. Entscheidend ist dabei, dass Strukturen sowohl Handeln produzieren als auch selbst durch das Handeln reproduziert werden eine These, auf die GidDENS (1997: 78) mit dem Begriff der «Dualität von Struktur» aufmerksam macht (Abbildung 1). In dem Prozess der Strukturation entscheiden die Akteure über die Kontinuität oder Veränderung von Strukturen und beeinflussen dadurch die Reproduktion sozialer Systeme und Interaktionszusammenhänge. Konsequenterweise müssen wir davon ausgehen, dass Akteure, die sich in ihrer Lebensrealität mit einer Fülle von zum Teil einander ausschließenden strukturellen Vorgaben konfrontiert finden, aus dieser Fülle dasjenige herausgreifen werden, das ihnen am besten zu ihrer Lebenssituation zu passen scheint. Dabei kann es auch zu einer Kombination einzelner Elemente aus verschiedenen Strukturvorgaben kommen, die dann als «neue Struktur» fortbesteht.

Der Prozess der Strukturation verlangt von den Akteuren ein von Fall zu Fall unterschiedliches $\mathrm{Ma} ß$ an Reflexion. Zu Zeiten gesellschaftlicher Kontinuität

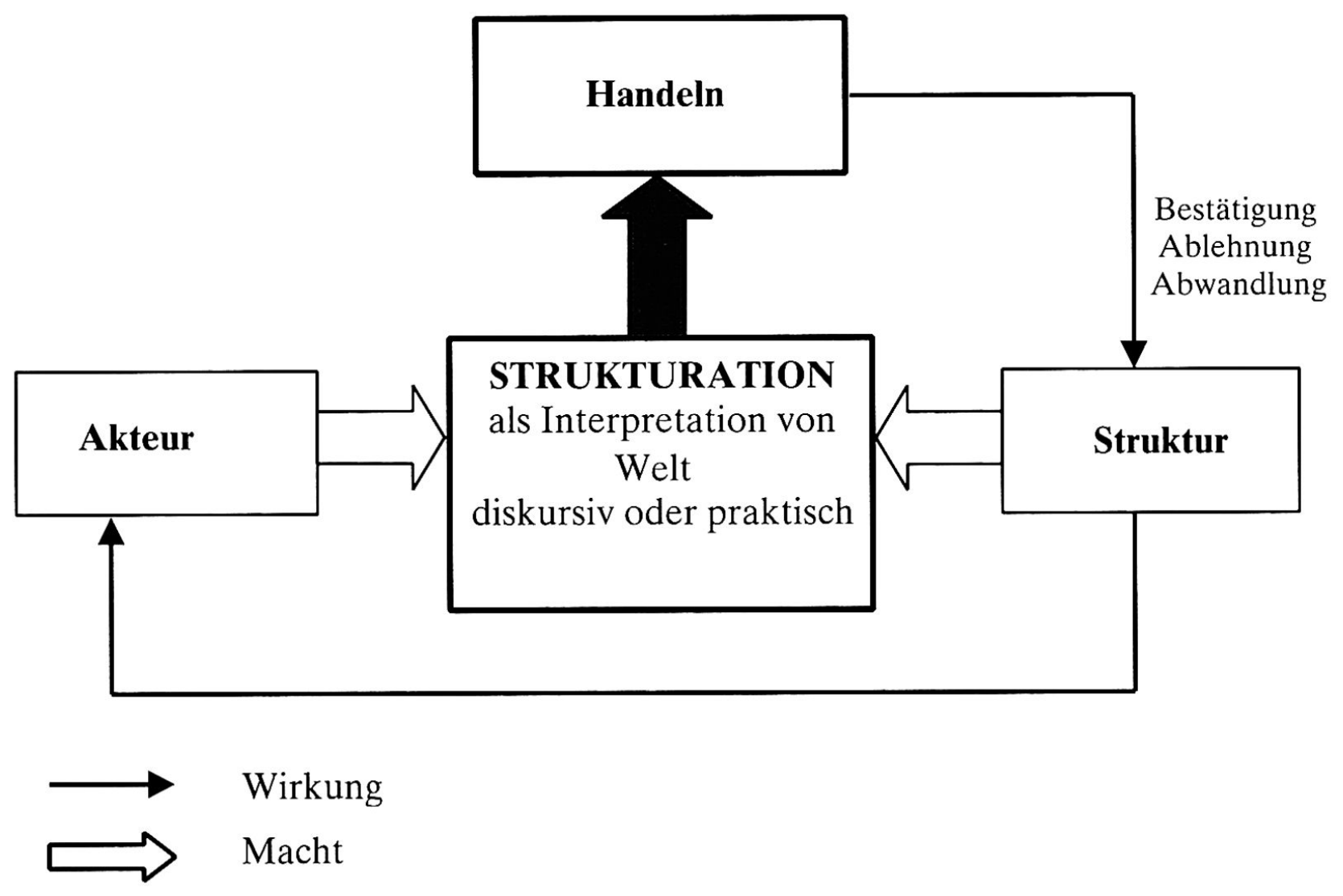

Abb. 1: Der Prozess der Strukturation - Vermittlungsfeld zwischen «Handeln» und «Struktur»

The process of structuration - Intermediary between "action» and "structure»

Le processus de structuration - Champ de médiation entre "action» et "structure»

Entwurf: S. TRÖGER, nach GIDDENS 1997: 51-77 
können sich die Akteure in vielen Entscheidungssituationen auf die Vorgaben ihres «praktischen Bewusstseins» (GidDENS 1997) berufen, die es ihnen gestatten, sich innerhalb der Routine des gesellschaftlichen Lebens zurechtzufinden. Zu Zeiten gesellschaftlichen Umbruchs, bei dem sich der strukturelle Handlungsrahmen teilweise auflöst beziehungsweise sich Situationen struktureller Vielfalt und Widersprüchlichkeit einstellen, hält das praktische Bewusstsein bald keine gesellschaftlich standardisierten Antworten für den Entscheidungsprozess mehr bereit. Erst eine reflexivdiskursive Auseinandersetzung mit der Vielfalt des Vorgegebenen kann den Akteuren eine Handlungsleitlinie anbieten. Dem Prozess und dem Potential zur Neudefinition struktureller Vorgaben wird in dieser Situation des Umbruchs eine besondere Dynamik zuwachsen, die sich in einem vielschichtigen Muster struktureller Vorgaben, strukturierendem Handeln und sich neu formierender Strukturelemente artikuliert.

\section{Tansania, ein Beispiel struktureller Heterogenität}

Wenn wir uns nun der Fallstudie aus Tansania zuwenden, richten wir unsere Aufmerksamkeit auf eine Region, die sich im Verlauf der letzten etwa dreißig Jahre mit einer Fülle der unterschiedlichsten und auch widersprüchlichsten Vorgaben konfrontiert fand. Das heißt, die Akteure, deren Handeln wir mit Bezug auf die Frage der Verwundbarkeit und sozialen Sicherung beobachten und analysieren, werden sich nicht auf eine Handlungsgewissheit praktischer Bewusstseinsinhalte verlassen können. Der in ihrem Handeln offenbarte Strukturationsprozess wird damit Einblicke in die Bedingungen der Wahl zwischen den Vorgaben und damit auch in die Freiheitsgrade des Handelns gewähren. Das zentrale Erkenntnisinteresse liegt dabei stets auf den Folgewirkungen dieses Handelns auf die Ernährungssituation der Haushalte, speziell derjenigen von verwundbaren Gruppen.

Schließen wir uns der oben angeführten These einer notwendigen Kongruenz zwischen Regelvorgaben und individuellen Handlungsmotiven bei Prozessen gesellschaftlicher Umverteilung an, dann werden wir bei der Analyse der sozialen Sicherung in der Untersuchungsregion unsere Aufmerksamkeit besonders auf die Nahtstelle zwischen den subjektiven oder auch intersubjektiv geteilten Bedürfnissen der betrachteten Menschen auf der einen und den gesellschaftlich etablierten Regulierungen des Teilens auf der anderen Seite richten müssen. Es stellt sich die Frage, wie sich der Wandel der Bedürfnislage der Handelnden unter dem Eindruck der strukturellen Vielfältigkeit und Widersprüchlichkeit in der Untersuchungsregion in den Erscheinungsformen gesell- schaftlicher Umverteilung niederschlägt und dadurch auch einen Wandel der normierten Regulierungen des Teilens nach sich zieht, der seinerseits dann auf das Zusammenleben der Menschen allgemein und speziell deren Verwundbarkeit durch Nahrungsdefizite zurückwirkt.

\section{Die Fallstudie}

\subsection{Ausgangsthesen und methodisches Vorgehen} "Mwatwima chani? - Awe, inzala!» - «Was hast du gegessen? Nichts, ich habe Hunger!» - ist eine übliche Begrüßungsformel für die Menschen, deren Lebenswelt wir in der Fallstudie betrachten. Der Gruß kennzeichnet in seinem alltäglichen Gebrauch entgegen seinem Wortlaut keine konkrete Notsituation. Er bedeutet im übertragenen Sinne in etwa: «Wie geht es dir? - Gut!» Das heißt, die Bewohner des UfipaPlateaus im Südwesten Tansanias (Abbildung 2) leben in einer Situation, in der eine Sorge um Nahrungssicherheit so zum Alltag gehört, dass sie sich in der Selbstverständlichkeit eines Grußes niederschlägt.

In welcher Weise eine solche Lebensperspektive für die Menschen handlungsleitend wird und sich in verschiedenen Lebensbereichen artikuliert, war Gegenstand einer dreieinhalbjährigen Feldforschung (1992-1996) bei insgesamt 385 Haushalten in acht Dörfern. Die Auswahl der Dörfer folgte einem Profil der Marktintegration von der Peripherie bis zur Nähe der Provinzhauptstadt Sumbawanga. Diese Differenzierung gründete auf der These, dass sich mit dem Grad ihrer Marktintegration die Bewohner der Dörfer unterschiedlich stark mit modernen Strukturen wie zum Beispiel Vermarktungsoptionen, Konsumanreizen, Monetarisierung und allgemein nicht-indigenen Werthaltungen konfrontiert finden. Das Ergebnis der Strukturation wird, so die weiterführende Überlegung, dann bei ansonsten ähnlichen Grundbedingungen in den Dörfern unterschiedlich ausfallen und auch unterschiedlich auf die Ernährungssicherheit von Haushalten wirken.

Die Ergebnisse, die in der Fallstudie vorgestellt werden, basieren auf Untersuchungen in einer peripheren Siedlung (Mtuntumbe) und einer marktintegrierten Siedlung (Mkima) bei insgesamt 162 Haushalten. Sie werden von weiteren Beobachtungen ergänzt (4.5.1) und wurden in ihrer Repräsentativität in den weiteren sechs Untersuchungsdörfern überprüft. Es wurde mit quantitativen Methoden, mit der Methode der teilnehmenden Beobachtung und mit partizipativen Methoden (PRA) gearbeitet (TRÖGER 1997, 2002). Die Verlässlichkeit der Ergebnisse wurde nach dem Prinzip der «mehrperspektivischen Triangulation» (KöCKEISSTANGL 1980: 363) sichergestellt. 


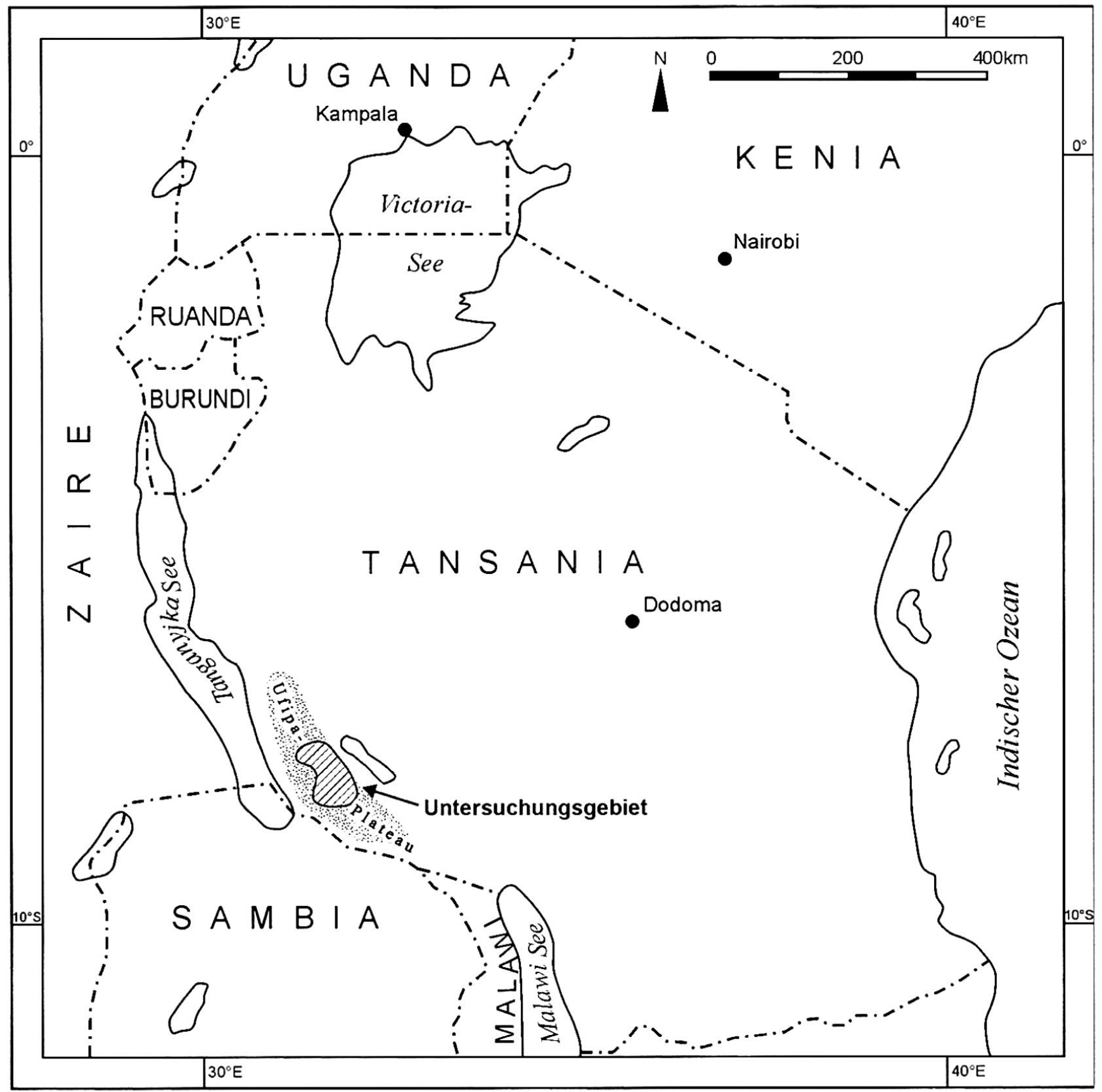

Abb. 2: Das Ufipa-Plateau im Südwesten Tansanias The Ufipa-Plateau in the southwest of Tanzania Le plateau d'Ufipa dans le sud-ouest de la Tanzanie Quelle: Entwurf: S. TRöGER; Kartengrundlage: Diercke Weltatlas 1987:136 (Abdruck mit freundlicher Genehmigung)

4.2 Die Problemstellung im Erhebungszeitraum Die «alltägliche Krise» einer tendenziellen Nahrungsunsicherheit erfährt dann eine Zuspitzung, wenn eine Dürreperiode Produktionseinbrüche zur Folge hat. Eine solche Situation finden wir in der Untersuchungsregion in dem Zeitraum von September 1994 bis März 1995 vor, als nach einer Dürre in der Anbauperiode 1993/94, der bereits zwei relativ schlechte Erntejahre vorausgegangen waren, die Haushalte unter Nahrungs- defiziten leiden. Ende September beklagen bereits $52 \%$ der Haushaltsvorstände in der marktintegrierten Siedlung und 23\% derer in der Peripherie ihre akute Mangelsituation, die nach ihren Angaben zum Teil schon seit einiger Zeit andauert (Abbildung 3). Für die nahe Zukunft bis zum Ende der Regenperiode im April sehen die Vorstände von $89 \%$ der Haushalte mit Marktanschluss gegenüber $57 \%$ in der Peripherie Probleme mit «Hunger» voraus. In der privilegierten 


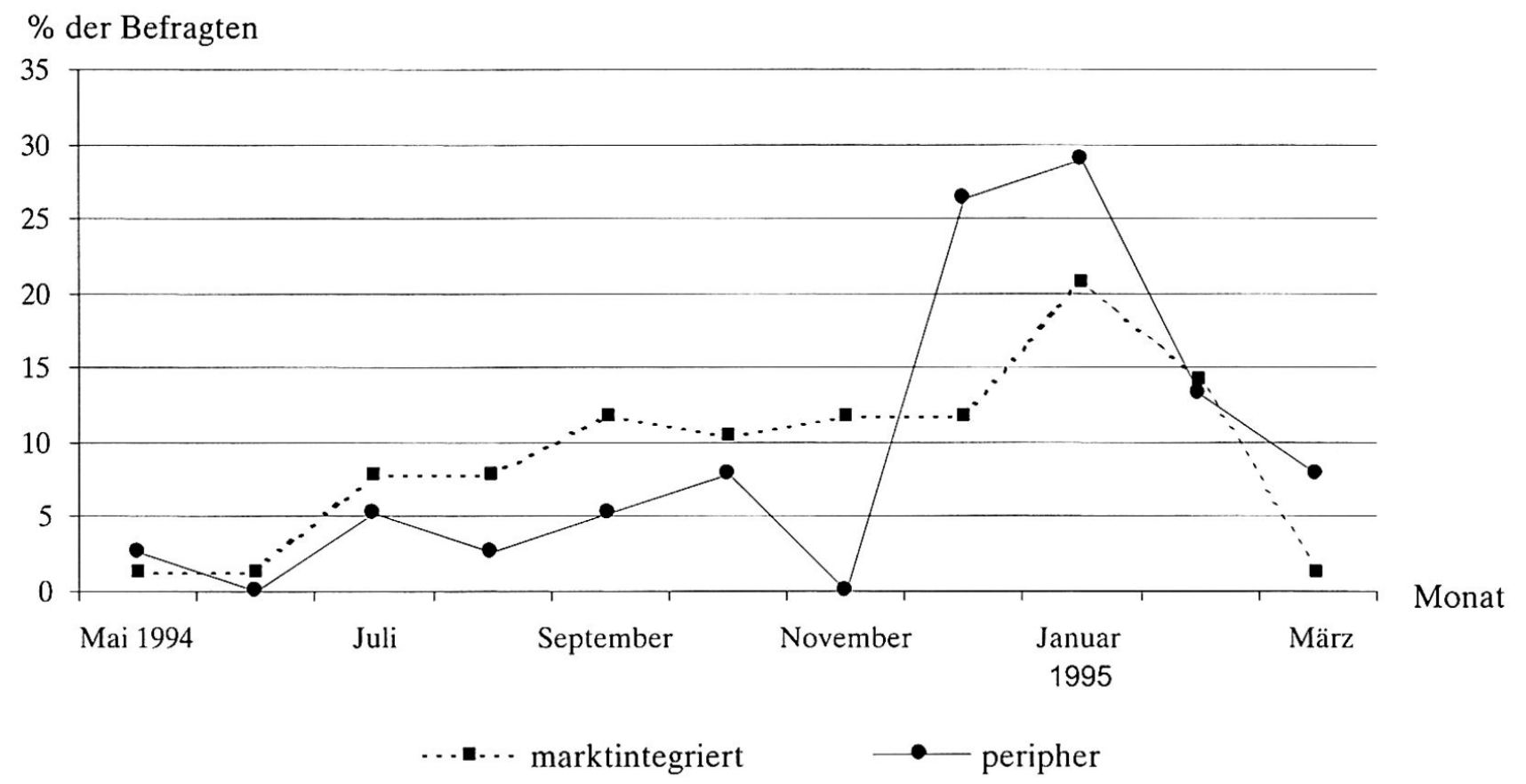

Abb. 3: Beginn des Hungers in marktintegrierter Lage und in der Peripherie The beginnings of hunger in market-integrated locations and the periphery Le début de la faim dans un lieu intégré à l'économie de marché et à la périphérie Quelle: Erhebung S. TRÖGER, September bis Oktober 1994

Rolle eines «Voyeurs» (SHIPTON 1990: 380) beobachten wir vor diesem Hintergrund die Wirksamkeit kultureller Regulierungen und Werthaltungen oder auch deren Versagen in dieser bedrängten Situation.

«Hunger» ist nicht gleich "Hunger»! - Die angegebenen Prozentwerte des «Hungers» legen jedoch für sich noch kein befriedigendes Zeugnis von der Situation der Bedrohung ab. Unsere Fragen nach Nsala «Hunger»- erweisen sich als mehrdeutig. «Hunger», das ist absoluter Nahrungsmangel - oder aber ein Mais-Defizit zur Zubereitung von Insima, dem Maisbrei und Hauptbestandteil des «vollkommenen Mahls» (SPITTLER 1993: 196). Die Menschen in marktintegrierter Lage kennzeichnen zu 97\% mit «Hunger» einen absoluten Nahrungsmangel, ihre Nachbarn in der Peripherie dagegen mehrheitlich, nämlich zu 54\%, ihren Mangel an Mais für Insima. Ein Vergleich dieser regional-semantischen Differenzierung mit der prozentualen Häufigkeit der Klage über «Hunger» unterstreicht die Brisanz der Mangelsituation in der marktintegrierten Siedlung.

\subsection{Ernährungssicherung im Zeichen kollektiver Arbeitsethik: Merkmale des indigenen Gesellschaftssystems}

«Als ich jung war, haben die Leute hart gearbeitet, nicht wie heute, wo sie sich nicht mehr darum bemühen, ihre Familie zu ernähren. Wenn jemand wirklich für seine
Mahlzeiten auf dem Feld anderer arbeiten musste, wurde er als faul angesehen. Damals haben sich die Menschen geschämt, wenn sie wegen Nahrungsmitteln für andere arbeiten mussten» (EsTher Kimitı, 83 Jahre).

Das indigene Gesellschaftssystem ruhte auf zwei Eckpfeilern - dem Land und der Arbeit, wobei das Land als die Basis des Lebens und die Arbeit als das Mittel des Lebens galten. Zur Systemstabilisierung trug ein Verständnis bei, das mit dem Land die Aufgabe eines Schutzes gegenwärtiger und zukünftiger Generationen und mit der Arbeit den Schutz des Überlebens durch Ernährungssicherheit verband. Da die Dorfgesellschaften bereits seit alters deutlich sozial differenziert waren und einige reichere Haushalte einer größeren Gruppe von Haushalten gegenüberstanden, deren Ernährungssicherheit saisonal oder auch periodisch bedroht war, gewährleisteten spezifische und über eine von allen anerkannte Moral abgesicherte Mechanismen der Umverteilung den gesellschaftlichen Fortbestand. Ein strenges Kontrollsystem, das sich zum Teil gefürchteter Sanktionen wie des Hexenfluchs bediente und das auf ein regelkonformes, an praktischen Bewusstseinsinhalten orientiertes und nicht diskursives Handeln der Akteure angelegt war, schützte vor Systemstörungen.

Die Kontrollmechanismen wirkten einer Destabilisierung in zwei Richtungen entgegen. Auf der einen Seite 
behinderten sie eine Überbeanspruchung der Solidarpflicht, indem sie die Mühe um Ernährungssicherheit zur höchsten Pflicht jedes einzelnen erklärten. Da man davon überzeugt war, dass Arbeitsbereitschaft eine Frage der Veranlagung und nicht der Erziehung sei - so gibt es «gute», arbeitswillige und «schlechte», arbeitsunwillige Kinder: itambi zisuma und itambi ziipe - sah die gesellschaftliche Kontrolle Maßnahmen vor, jedes Mitglied der Gemeinschaft zu einem solchen Arbeitseinsatz zu veranlassen, der seine eigene Ernährungssicherheit garantierte. Hilfsleistungen für so wahrgenommene «faule», nicht ausreichend arbeitende Mitmenschen wurden verweigert. Das heißt, das Problem der Ernährungsunsicherheit wurde in einem Umkehrschluss zunächst den Betroffenen selbst angelastet und damit von jenen Problemlagen abgegrenzt, die eine Hilfe durch die anderen erforderten. Mit Abstrakta wie «Scham» und «Faulheit» wurde das Arbeits-Handeln in Bahnen gelenkt, die dem Erhalt der Gemeinschaft zuträglich waren. Erschien auf Seiten der Bedürftigen die Bedingung größtmöglichen Arbeitseinsatzes zur Ernährungssicherung dagegen erfüllt, verlangten dann die Regeln der Gemeinschaft eine Umverteilung der Überschüsse der reicheren Haushalte. Diese Solidarpflicht war zum Schutz der Bedürftigen ihrerseits gesellschaftlich mittels normierter Sanktionen einschließlich der Bedrohung durch Hexenfluch abgesichert, über deren Anwendung die «elders» in ihrer Autoritätsfunktion wachten.

Wenn wir uns im Folgenden der Ausprägung sozialer Sicherung in der Gegenwart zuwenden, konzentrieren wir uns auf solche Mechanismen, die der kollektiven Ernährungssicherung zur Stabilisierung der Gemeinschaft als Ganzer dienten (andere Aspekte sozialer Sicherung wie die Systeme reziproken Austauschs und neue Formen von Solidarverbindlichkeiten werden an anderer Stelle beschrieben und analysiert - TRÖGER 2002). Im Zusammenhang mit jener Absicherung der Gemeinschaft ist an erster Stelle die Verpflichtung für reichere Haushalte zu nennen, ihren ernährungsbedrohten Nachbarn Arbeit auf ihren Feldern anzubieten. Für eine solche Arbeit erhielt der Hilfesuchende als Gegenwert für einen Arbeitstag angestammterweise ein debe Getreide - einen Korbmaß von etwa $20 \mathrm{~kg}$. Die Getreidemenge entsprach dem Tagesoder sogar Zweitages-Bedarf größerer Haushalte. Sie erfüllte so das Ziel der Ernährungssicherung und wird im Folgenden in Abgrenzung zur heutigen «Lohnarbeit» als «Überlebensarbeit» gekennzeichnet (4.5.3).

Als weitere Maßnahmen der Systemstabilisierung sind solche zu nennen, die speziell auf die Absicherung in der Zukunft zielten. Es galt, die heranwachsende Generation und mit ihr die eigene Versorgung im Alter zu schützen. Besonders strenge und bei einer Verweigerung mit sozialer Ächtung geahndete Regeln, die
Kleinkinder in den Schutz aller stellten und jedem Haushalt eine Mitversorgung der Nachbarskinder im Bedarfsfall auferlegten, erschienen geeignet, den Fortbestand in der Zukunft zu gewährleisten. Damit sich keiner dieser Pflicht entziehen konnte, war es verpönt, hinter verschlossenen Türen zu essen, und nicht jedem Kind entsprechend seines Alters beim Essen behilflich zu sein.

\subsection{Die Dorfgesellschaften im Fadenkreuz des strukturellen Wandels}

Die Einflusskraft der neuen, seit der zweiten Hälfte der 60er Jahre in den Dorfgesellschaften wirksamen Strukturen, richtet sich auf die benannten Mittelpunkte des angestammten Gesellschaftssystems. Die Entwicklung nimmt ihren Ausgang bei der Erfahrung einer im Rahmen der Arusha-Erklärung 1967 propagierten Entmachtung angestammter Autoritäten und der durch sie repräsentierten Regulierungen und Überzeugungen, wobei die Entmachtung nicht allein durch die nationale Gesetzgebung durchgesetzt, sondern für die Menschen auch in der Missachtung ihrer etablierten Zugangsrechte zu Land durch die eigenen politischen Führer während der Umsiedlung greifbar wird. Im Zuge der Umsiedlung - «operation ujamaa» im Sprachgebrauch - müssen dann 1974 Tausende und Abertausende Menschen allein auf dem Plateau ihre angestammten Siedlungsgemeinschaften verlassen und sich in vom Staat festgelegten «Mittelpunktsdörfern» in der Nachbarschaft von ihnen fremden Menschen ansiedeln. In Ergänzung zu diesem für viele beängstigenden Einschnitt in die Grundfesten ihres Lebens werden im Anschluss an die Umsiedlung in rascher Folge und zum Teil zeitlicher Überlappung strukturelle Einflüsse wie die Marktintegration mit den mit ihr verbundenen Maßnahmen der Mais-Produktionsförderung, die fortschreitende Monetarisierung in Verbindung mit erweiterten Konsummöglichkeiten, die Rücknahme staatlicher Subventionen im Zuge der Strukturanpassung und im Zusammenhang mit Landzugangs-Fragen nationale Reglementierungen wie das neue Landrecht in der Region wirksam.

\subsection{Soziale Sicherung im Zeichen des gesellschaft- lichen Wandels: die Situation der 90er Jahre}

\subsubsection{Verpflichtet die Not des anderen zum Teilen?}

Die Armut des Nachbarn - im Jahresverlauf nahrungsunsichere Haushalte gelten als «arm»- ist allgemein in der Wahrnehmung der Menschen kein hinreichender Grund für eine gesellschaftliche Umverteilung. Erst die von der Gemeinschaft so interpretierte jeweilige Ursache der Armut legt das gesellschaftlich anerkannte Maß und die Art der Hilfe fest. Den «Faulen» wird nicht geholfen. Allerdings wird es heute immer schwieriger, an den überkommenen Bewertungsindikatoren für «Faulheit» und «Fleiß» festzuhalten. Ein 


\section{$\%$ der Befragten}

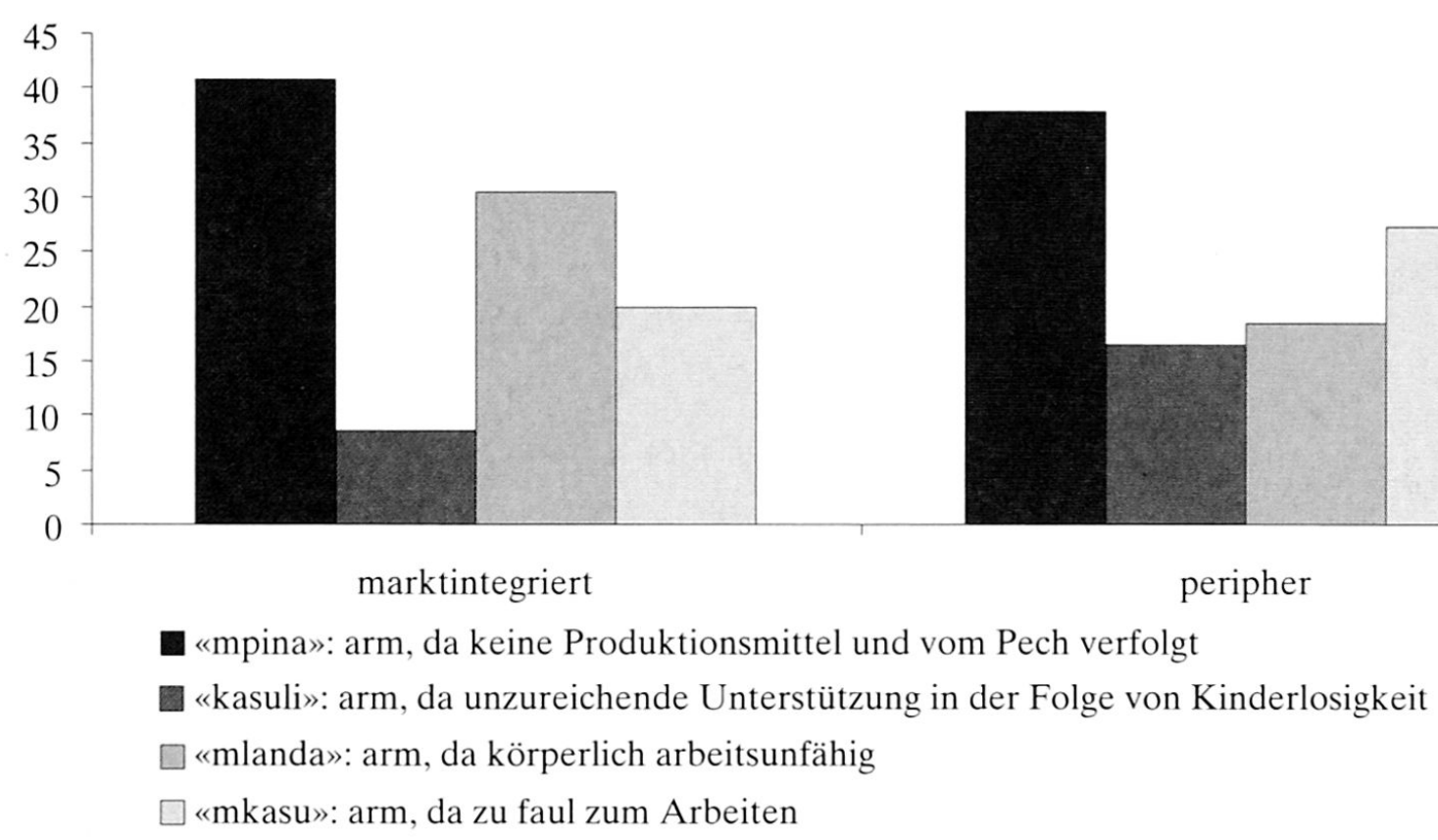

Abb. 4: Gründe für chronische Nahrungsunsicherheit in der Interpretation der Mitmenschen - der regionalhistorische Vergleich

Reasons for chronic food insecurity in the perception of the neighbours - a regional-historical comparison

Raisons pour l'insécurité chronique alimentaire dans la perception des voisins-la comparaison historico-régionale Quelle: Erhebung S. TröGER (in Zusammenarbeit mit IFSP/GTZ Sumbawanga), September bis November 1995

leerer Speicher kann auf einen geringen Arbeitseinsatz hinweisen. Er kann jedoch auch in Verbindung mit der Verarmung des Haushalts stehen, der sich gezwungen sah, die gesamte Produktion zu verkaufen, um eine existentielle Notlage zu bewältigen. Insgesamt macht sich mit zunehmender Marktintegration die Erfahrung breit, dass die einfache Gleichung, nach der ein hoher Arbeitseinsatz mit einer Situation der Ernährungssicherheit belohnt wird, keine Gültigkeit mehr hat. Aus diesem Grund sind die Menschen mit der Erfahrung einer Markteinbindung vorsichtiger, ihre Mitmenschen für ihre Notlage direkt verantwortlich zu machen und als «faul» abzuurteilen (Abbildung 4). Mit dieser Verunsicherung verliert dabei aber die angestammte Interpretationsregel, nach der die Menschen ihre Solidarverpflichtung bestätigt fanden und auf der anderen Seite die Bedürftigen ihr Recht auf Hilfe ableiteten, an Durchsetzungskraft.

Armutssituationen werden mit zunehmender Marktintegration in den 90er Jahren vorrangig auf einen Mangel an Produktionsmitteln wie Land, Arbeitskraft und Ochsenpflugkraft zurückgeführt. Ob und in welcher Weise der dargestellte Solidarmechanismus eines Arbeitsangebots für solcherart arme Mitmenschen heute (noch) angesichts des strukturellen Wandels zur sozialen Sicherung nahrungsgefährdeter Haushalte beiträgt, werden die Beobachtungen in den Siedlungen verdeutlichen.

\subsubsection{Verpflichtet Reichtum zum Teilen?}

Eine wichtige Voraussetzung für das Funktionieren des Umverteilungssystems war eine Transparenz im Hinblick auf die Verfügbarkeit von Vorratsmengen und Viehbestand, auf der Basis derer eine Verpflichtung zum Teilen glaubhaft abgeleitet werden konnte. Diese Transparenz ist mit den modernen Entwicklungen - allen voran der Monetarisierung und dem Transportanschluss - nicht mehr gegeben. Ein großer Teil der Maisproduktion wird bald nach der Ernte verkauft. Hilfsverpflichtungen gegenüber anderen Dorfbewohnern lassen sich anschließend leicht mit dem dann leeren Getreidespeicher begründet ablehnen. Bargeld kann dagegen im Haus vor den abschätzenden Blikken der Nachbarschaft verborgen werden und kann so kaum einen Appell an Solidarverpflichtungen stützen.

Doch nicht allein die verminderte Transparenz innerhalb der Gemeinschaft bewirkt die Schwächung der Umverteilungsmechanismen. Der im Rahmen der Uja- 
maa-Politik national angeordnete Abbau angestammter Autoritätsstrukturen und deren gesellschaftlicher Legitimität hat dazu beigetragen, dass ein Teilen für die Reicheren in den 90er Jahren keinen Anreiz mehr birgt. Dörfliche Autoritätsstrukturen und -zuweisungen sind nun weitestgehend von der Frage sozialen Aufstiegs abgekoppelt und werden an nationalen Interessen ausgerichtet. Mit dem Abbau angestammter Autoritätsstrukturen geht dann auch eine Schwächung von Sanktionen wie dem Hexenfluch einher, die früher der gesellschaftlichen Umverteilung zur Durchsetzung verhalfen.

\subsubsection{Zur Artikulation gesellschaftlicher Umvertei- lung in den Gemeinschaften der 90er Jahre}

Betrachten wir zunächst die Entwicklung gesellschaftlicher Umverteilung im Zusammenhang mit dem in vielen Regionen Afrikas verbreiteten Angebot der «Überlebensarbeit». Hier stellt sich die Brisanz der Umverteilungs-Problematik zum einen in dem Wechselspiel zwischen Bedarf und Angebot dar. Während für «normale» Jahre lediglich $12 \%$ der befragten Haushalte in der marktintegrierten Siedlung und $15 \%$ in der Peripherie auf ihren Bedarf an einem Zu-Verdienst durch «Überlebensarbeit» hinweisen, geben zu Beginn der Hungersaison 94/95 34\% beziehungsweise 29\% der Befragten an, ihr Haushalt hinge in seinem Fortbestand von einem solchen $\mathrm{Zu}$-Verdienst ab. In Notzeiten gründen somit im Durchschnitt etwa ein Drittel der Haushalte in den Siedlungen ihre Krisenbewältigung auf die Überschüsse anderer, die ihnen in Form von Arbeit angeboten werden können.

Doch nicht alle finden auch Arbeit. Wie weit der Hunger tatsächlich mit einer «Überlebensarbeit» zu mildern ist, variiert mit dem Grad der Marktintegration der Notleidenden. Dies zeigt sich am deutlichsten in dem Monat Februar, wenn produktionstechnisch es ist die Zeit nach der zweiten Unkrauthacke - der Bedarf an Arbeitskräften gering, haushaltstechnisch in dieser letzten Konsumphase vor der Ernte der Bedarf an «Überlebensarbeit» jedoch besonders hoch ist. Mit der Anzahl der Arbeitsmöglichkeiten sowie der Art und Höhe der Entlohnung entscheiden zwei Variablen über den Erfolg der Umverteilung.

Nach unseren Erkenntnissen wird dementsprechend in der Siedlung mit peripherer Lage das Arbeitsangebot dann zu größeren Anteilen von den wohlhabenderen Haushalten bestritten, wenn saisonal bedingte Nahrungsengpässe auch die Haushalte der Verwandten ergriffen haben. In diesem Moment bestimmt die Moralbindung reicherer Haushalte und nicht mehr die Familiensolidarität das Bild der gesellschaftlichen Umverteilung und lässt die Reicheren zusätzliche Arbeitsmöglichkeiten einrichten. Die Situation in der marktintegrierten Siedlung stellt sich dagegen mit deut- licher Akzentverschiebung dar. Hier ist der Arbeitsmarkt maßgeblich von dem ökonomischen Kalkül der Geberseite geprägt. Das Angebot verringert sich - und kommt in Extremfällen völlig zum Erliegen - wenn der Wachstumszyklus der Feldpflanzen im Februar eine solche Reduktion des Arbeitseinsatzes gestattet und wenn auch die wohlhabenderen Haushalte selbst eine Notwendigkeit zur Sparsamkeit empfinden. $42 \%$ der auf Überlebensarbeit hoffenden nahrungsunsicheren Haushalte finden in dieser Zeit keine Arbeit. Während sich also das Arbeitsangebot in der Peripherie offensichtlich mehr an dem Bedarf der nahrungsunsicheren Haushalte orientiert und ein Gefühl der Solidarverpflichtung reichere Haushalte dann einspringen lässt, wenn die Hilfskapazität der Nachbarn und nahen Verwandten überfordert ist, werden solche Engpässe im Zeichen des Marktanschlusses nicht oder nur dann aufgefangen, wenn es sich ökonomisch auszahlt.

Den im Arbeitsangebot offensichtlichen Übergang von einer Arbeit auf dem Feld anderer als «Überlebensarbeit» zur «Lohnarbeit» spiegelt auch die Höhe und Art der Bezahlung der Arbeit im regionalen Vergleich. Die Entlohnung stellt sich in einer dreifachen Abstufung dar. In der Peripherie kommt das angestammte Entlohnungsmaß von 1 debe noch in einigen Fällen und speziell im Kontext verwandtschaftlicher Beziehungen zur Anwendung. Das Normalmaß ist hier allerdings auch bereits die an dem jeweils aktuellen Marktpreis orientierte Entlohnung mit Mais. Da in der Peripherie der Marktwert von Mais nicht so hoch ist wie in Markt- und Stadtnähe, erhalten die Arbeitenden jedoch vergleichsweise mehr Getreide für ihre Arbeit. Häufig werden sie noch mit Naturalien statt mit Geld ausgezahlt und laufen so nicht Gefahr, in dieser Mangelzeit für ihr Geld keinen Mais zu erhalten.

In der marktintegrierten Siedlung lassen sich die reicheren Bauern auf solche Vergütungen praktisch nicht mehr ein. Das frühere Überlebensmaß 1 debe findet gar keine Anwendung, und selbst eine Entlohnung in Naturalien stellt einen Ausnahmefall dar. Die Arbeit auf dem Feld anderer hat hier praktisch völlig den Charakter von Lohnarbeit angenommen, deren Vergütung nicht mehr an dem Bedarf der Arbeitsuchenden, sondern an den ökonomischen Interessen des Arbeitgebers orientiert ist und so besonders zu Spitzenzeiten des Mangels bei weitem nicht mehr den ÜberlebensBedarf nahrungsunsicherer Haushalte deckt.

Im Zusammenhang mit dem zweiten Aspekt gesellschaftlicher Umverteilung zum Schutz der Gemeinschaft, nämlich der Ernährungssicherung für Kleinkinder, sind die Merkmale des Wandels subtiler und nur über eine Teilnahme am Dorfleben zu erschließen. Im Verlauf unserer Dorfaufenthalte in der peripheren 


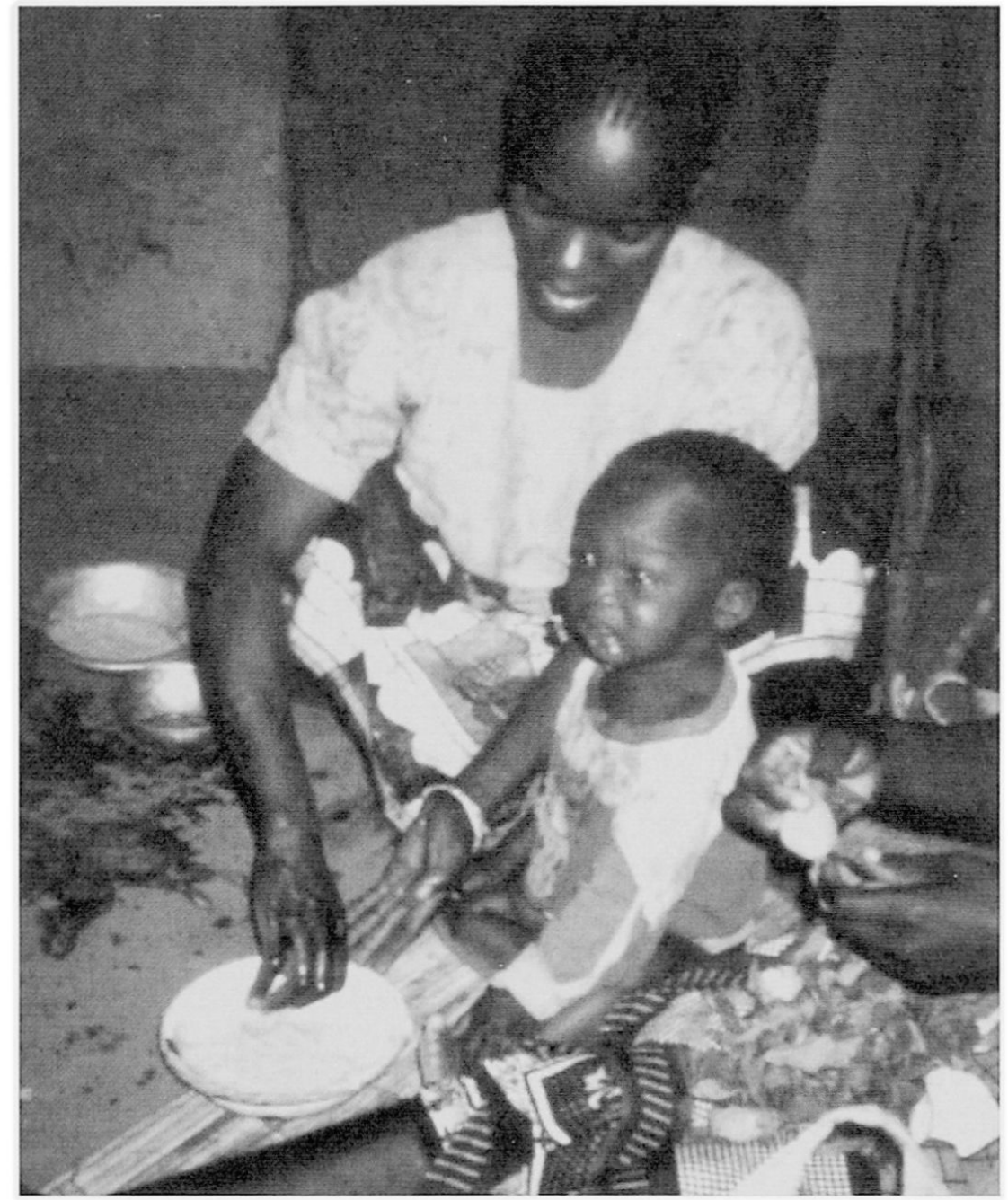

Abb. 5: Mama Esther hilft beim Essen

Foto: S. TRÖGER

MAMA Esther helps feeding

MAMAN Esther aide au repas

Siedlung können wir in diesem Zusammenhang keine Abkehr von den angestammten Regeln des gesellschaftlichen Wohlverhaltens feststellen. Die einzige Beobachtung, die einen auch hier in Zukunft einsetzenden Wandel vorankündigen mag, sind die häufigen Diskussionen über die Notwendigkeit aber auch Belastungen einer solchen Mitversorgung der Nachbarskinder.

In der Siedlung mit Marktanschluss häufen sich dagegen die Verstöße gegen die Regeln. Die Maßnahmen zur Abwehr hungriger Nachbarskinder reichen von dem Verbergen der Mahlzeit durch das Schließen der Türe über eine zeitliche Verschiebung der Mahlzeit in die Zeit der Nachtruhe der Kinder bis zu der Verweigerung von Hilfe für - in dem beobachteten Fall - ein dreijähriges Kind, dessen kleine und noch hitzeempfindliche Finger keine Stücke aus dem heißen Insima-Berg heraustrennen können (Abbildung 5). Im
Verlauf der schnell eingenommenen Mahlzeit gelingt es dem Kind nicht, sich eine ihm angemessen große Menge Maisbrei zu sichern.

Die Abwehr der Kinder wird mit dem Hinweis auf ein Fehlverhalten der Mütter legitimiert. Die Nachbarfrauen leiten die Berechtigung ihrer Ablehnung der Kinder aus den angeblich häufigen vormittägigen Barbesuchen der Kindsmutter, der Vernachlässigung der Kinder in der Folge einseitigen Gewinnstrebens der Mutter, das sich in übermäßiger Feldarbeit zeigt, oder dem geplant-absichtlichen Zurücklassen der hungrigen Kinder zur Essenszeit ab. Auch die überkommene Furcht vor Hexenfluch dient der Legitimation einer Hilfs-Verweigerung. Mit dem Hinweis darauf, eine Erkrankung des Kindes könne mit einer HexenAnschuldigung (Vergiften) quittiert werden, wird dem Kind eine Teilnahme an der Mahlzeit verwehrt. 


\section{Gewinner und Verlierer des gesellschaftlichen Wandels}

«Heute hilft man, wenn man will. Früher war das ein 〈Muss). Man hilft, wenn man Mitleid empfindet» (FIDELI NDolezI, 60 Jahre).

Der Ausgangspunkt der Analyse war die These, dass die angesprochenen Regeln gesellschaftlicher Umverteilung dem Schutz der Gesamtheit und des gesellschaftlichen Fortbestands dienten. Mit dem Grad der Marktintegration und «Modernisierung» der Menschen können wir heute eine Schwächung solcher Mechanismen feststellen. Während in der Peripherie (noch) eine Moralbindung wirksam ist, die dem angestammten Mechanismus der Umverteilung gehorcht, gerät mit fortschreitender Marktintegration die Gesellschaft als Ganze immer mehr aus dem Blick. Das Handeln orientiert sich an Partikularinteressen, die bestenfalls noch den Zusammenhalt der engeren Familie ins Auge fassen. Phänomene wie eine Marktangleichung der Arbeitsentlohnung, Geber orientierte Regulierungen des Arbeitsmarktes, das Verschließen der Türen zur Essenszeit sowie die Umkehrung der Selbstverschuldungs-Klausel sind Indikatoren für einen allgemeinen Niedergang der sozialen Sicherheit durch Umverteilung, der in einer Bilanzierung mit Bezug auf die Frage der Ernährungssicherheit der Menschen nach einer Gewinner- und einer Verliererseite zu differenzieren ist.

Auf der Gewinnerseite werden in der marktintegrierten Siedlung all jene stehen, die über eine einigermaßen solide Ressourcenbasis verfügen und die sich nun nicht mehr zum Teilen gezwungen sehen, akkumulieren können und Hilfe nur nach eigenem Gutdünken leisten. Es befinden sich jedoch nicht nur die im Dorfkontext Reichen selbst auf der Gewinnerseite. Auch diejenigen, die zu deren engerer Familie zählen, werden von dem gesellschaftlichen Wandel profitieren. Als letzte Bastion der Umverteilung ist die Kernfamilie oder engere Familie geblieben. Auf die Hilfe innerhalb dieses Kreises kann man sich noch immer weitestgehend verlassen, und so werden die Angehörigen der reicheren Dorfbewohner von der Einschränkung der allgemeinen Umverteilung profitieren, da sie nun zu dem kleineren Kreis derjenigen zählen, die bei einer Verteilung von Überschüssen berücksichtigt werden.

Auf der Seite der Verlierer dagegen können wir die überwiegende Mehrzahl der Menschen in den Dörfern vermuten, beklagen doch über zwei Drittel der Haushalte eine zumindest periodische Nahrungsunsicherheit. Für diese ohnehin gefährdeten Haushalte müssen wir von einer tendenziellen Verschlechterung der Ernährungssituation ausgehen. Sie können sich nicht mehr auf die Solidarverpflichtung der Reichen beru- fen, und es bleibt ihnen lediglich die Hoffnung auf das Mitleidsgefühl, das den einen oder anderen aus der Gruppe der Wohlhabenderen zu einer Gabe veranlassen wird. Auf der anderen Seite sind jedoch auch die ärmeren Haushalte an die fortbestehende Hilfsverpflichtung innerhalb des engeren Familienkreises gebunden und müssen hier das wenige, das sie haben, mit ihren Familienangehörigen teilen. Eine Entwicklung in Richtung zunehmender Verarmung und Verwundbarkeit durch Nahrungsdefizite ist die vorhersehbare Folge und entspricht auch den Beobachtungen im Dorfzusammenhang. Da in dem peripheren ländlichen Raum auch keine diesbezügliche Entlastung durch formelle Sicherungssysteme - die in Tansania ohnehin unzureichend sind (KLEMP 1995) - zu erwarten ist, stellt sich die Entwicklung der Sozialen Sicherheit für etwa zwei Drittel der ländlichen Bevölkerung negativ dar. Die Menschen sind aufgerufen, sich selber neue Sicherungsstrukturen und -syteme zu schaffen, was ihnen auch unter bestimmten Voraussetzungen gelingt (TRÖGER 2002).

\section{Zur Dynamik des gesellschaftlichen Wandels}

Wurde bisher der Eindruck einer vergleichsweise stabilen Sicherungslage in der Peripherie vermittelt, so muss dieser relativiert werden. Bereits die Beobachtungen, dass auch in der Peripherie das Getreidemaß von 1 debe nur noch selten und dann zumeist bei der Entlohnung naher Verwandter Verwendung findet und dass die Frage einer Mitversorgung der Kleinkinder aus der Nachbarschaft mittlerweile zum Gegenstand der Diskussion geworden ist, deutet auf eine Ungleichzeitigkeit eines einheitlichen historischen Entwicklungswegs der Plateausiedlungen hin. Es ist zu vermuten, dass sich die heute noch vorrangig in den marktintegrierten Siedlungen anzutreffende Auflösung der innergesellschaftlichen Umverteilung im Sinne konzentrischer Kreise immer weiter in die Peripherie hinein ausbreiten wird. Da ähnliche Entwicklungen in Richtung Individualisierung und Schwächung angestammter Sicherungssysteme in ihrem Erscheinungsbild auch in anderen Regionen Afrikas zu beobachten sind - aus eigener Anschauung kennt die Autorin vergleichbare Prozesse in Malawi und Zimbabwe - ist es auch von übergreifendem Interesse, einen näheren Einblick in die hinter dem Wandel zu vermutenden Strukturationsprozesse zu nehmen.

Gehen wir davon aus, dass bisher die Menschen ihre Handlungsentscheidungen gemäß eines kollektiv verankerten Solidarbewusstseins trafen. Ihr Handeln wurde dabei zumeist von praktischen Bewusstseinsinhalten geleitet und bedurfte keines reflexiven Umgangs mit den Ansprüchen auf Seiten der Gemeinschaft. Doch nicht allein dieses Zusammenspiel von Erwar- 
tungen und Verpflichtungen ließ ihr solidarisches Handeln in den vorgezeichneten Bahnen verlaufen. Hinzu traten vielmehr noch Regulative wie Scham- und Schuldgefühle und auch kollektiv abgesicherte Kontrollmechanismen, die das Einhalten der normativen Vorgaben gewährleisteten.

Diese Handlungsgewissheit ist nun aufgehoben. Die Menschen finden sich mit Strukturen konfrontiert, die ihnen in der Zusammenschau die widersprüchlichsten Handlungsentscheidungen nahe legen. Angesichts solcher Gemengelage der verschiedensten Vorgaben von dem Ideal individuellen Gewinnstrebens über die Erfahrung miteinander konkurrierender Autoritäten und dadurch geschwächter Kontrollinstitutionen bis zu einer Verunsicherung im Hinblick auf das zu erwartende Handeln der Mitmenschen, die sich in gleicher Weise ihren Weg durch die Strukturenvielfalt bahnen müssen - erscheint ein Handeln gemäß anerkannter und gelernter Wege nicht mehr den Anforderungen der Lebensabsicherung zu entsprechen. Doch noch immer sind tief verankerte und sozial gelernte Mechanismen der Verpflichtung und Legitimierung wirksam. Diese Mechanismen erfahren in den Dorfgesellschaften nun eine Um-Funktionalisierung. Die «Selbstverschuldungs-Klausel», die ursprünglich dem Schutz der Gemeinschaft vor Schmarotzertum diente, wendet sich nun letztlich zum Beispiel gegen die Kleinkinder, deren Mütter des Müßiggangs und der Trunksucht bezichtigt werden. Der Hexenfluch, der zuvor demjenigen drohte, der seiner Solidarpflicht nicht nachkam, fungiert nun als Legitimation von Hilfsverweigerung. Nicht das Argument: «Ich habe selbst zu wenig», sondern vielmehr das so interpretierte Fehlverhalten der Mitmenschen wird zum Anlass genommen, zuvor verbindliche Solidarverpflichtungen abzuwehren, und es ist vor diesem Hintergrund ScHorr (1988: 104) zuzustimmen, der eine allgemeine Situation gegenseitigen Misstrauens für Fehlentwicklungen verantwortlich macht.

Insgesamt hat somit in das Zusammenleben der Menschen in den 90er Jahren eine tendenzielle Individualisierung der Alltagswelt-Interpretationen Einzug gehalten, die es den Akteuren gestattet, ihr Handeln von der Gemeinschaft unkontrolliert an dem Maßstab eigener individueller Interessen zu orientieren. In der Konsequenz steht es den Reichen weitestgehend offen, sich nach ihrem eigenen Gutdünken die Vorteile aus den angestammten Vorgaben herauszusuchen und sie beliebig, das heißt von der Gemeinschaft weitestgehend unkontrolliert, mit Versatzstücken der vielfältigen «neuen Strukturen» zu kombinieren. Den von Nahrungsdefiziten Betroffenen kann es jedoch gelingen, ihre dadurch zunächst tendenziell größere Verwundbarkeit herabzusetzen, indem sie auf der Basis eines reflexiven Umgangs mit dem gesellschaftli- chen Wandel und seiner sozialen Konsequenzen neue, angepasste Sicherungssysteme schaffen. $\mathrm{Zu}$ diesen zählen neue religiöse Gruppierungen und Solidargemeinschaften wie zum Beispiel Brau- und Spargruppen (TRÖGER 2002).

\section{Literatur}

Benda-Beckmann, F. von et al. (1988): Introduction: Between Kinship and the State. - In: Benda-BecKMANN, F. von et al. (Hrsg.): Between Kinship and the State: Social Security and Law in Developing Countries. - Dordrecht: Foris Publications: 7-20.

Bossert, A. (1988): Formal and informal social security - a case study of Tanzania. - In: BENDA-BECKMAN, F. von et al. (Hrsg.): Between Kinship and the State: Social Security and Law in Developing Countries. Dordrecht: Foris Publications: 211-228.

De Bruijn, M. \& H. van DijK (1994): A Pastoral Society in Crisis after the Droughts: Who Cares for Social Security? - In: Dittrich, S. \& J. Petersen-ThumSER (Hrsg.): Social Security in Africa - Proceedings of the International Conference held in Berlin, November 1993. - Berlin-Tegel: ZWS Zentralstelle für Wirtschafts- und Sozialentwicklung: 199-232.

DFID (Department for International Development) (1999): Sustainable Livelihoods Guidance Sheets. London.

Giddens, A. (1997): Die Konstitution der Gesellschaft. Grundzüge einer Theorie der Strukturierung. - 3. Auflage, Frankfurt am Main, New York: Campus Verlag. KASPERSON, R. (2001): Vulnerability and Global Environmental Change. - In: IHDP Newsletter of the International Human Dimensions Programme on Global Environmental Change (2001) 2:2-3.

Klemp, L. (1995): Soziale Sicherheit für Frauen in Tanzania - zwischen Tradition und Selbsthilfe. - In: NORD-SÜD aktuell 1: 83-90.

KöCKeis-Stangl, E. (1980): Methoden der Sozialisationsforschung. - In: HurrelmanN, K. \& D. Ulich (Hrsg.): Handbuch der Sozialisationsforschung. - Weinheim, Basel: Beltz: 321-370.

Partsch, M. (1983): Prinzipien und Formen sozialer Sicherung in nicht-industriellen Gesellschaften. - = Sozialpolitische Schriften 48, Berlin.

Platteau, J.P. (1991): Traditional Systems of Social Security and Hunger Insurance: Past Achievements and Modern Challenges. - In: Ahmad, E., Drèze, J., Hills, J. \& A. SEN (Hrsg.): Social Security in Developing Countries. - Oxford: Clarendon: 112-170.

Popkin, S.L. (1979): The Rational Peasant - The political economy of rural society in Vietnam. - Berkeley u.a.: University of California Press.

SchmidT, S. (1992): Social Security in Developing Countries: Basic Tenets and Fields of State Intervention. - In: Getubig, I.P. \& S. Schmidt (Hrsg.): Rethinking Social Security. Reaching out to the Poor. 
- Kuala Lumpur: Asian and Pacific Development Centre: $18-40$.

SchotT, R. (1988): Traditional Systems of Social Security and their Present-Day Crisis in West Africa. In: Benda-Beckman, F. von et al. (Hrsg.): Between Kinship and the State: Social Security and Law in Developing Countries. - Dordrecht: Foris Publications: 89-107.

Scorr, J.C. (1976): The Moral Economy of the Peasant. Rebellion and Subsistence in Southeast Asia. - New Haven, London: Yale University Press.

ShiPTON, P. (1990): African Famines and Food Security: Anthropological Perspectives. - In: Annual Review of Anthropology 19: 353-394.

SpITtLer, G. (1993): Lob des einfachen Mahles. Afrikanische und Europäische Eßkultur im Vergleich. In: Wierlacher, A., Neumann, G. \& H.J. Teuteberg (Hrsg.): Kulturthema Essen.-Berlin: Akademie Verlag: 193-210.

TRÖGER, S. (2002): Handeln zur Ernährungssicherung im Zeichen gesellschaftlichen Umbruchs. Untersuchungen auf dem Ufipa-Plateau im Südwesten Tansanias. - Studies in Development Geography, Saarbrücken: Breitenbach Verlag (im Druck).

TRÖGER, S. (1997): PRA - Ein Instrument der Feldforschung? Erfahrungen bei der Analyse von Ernährungsproblemen auf dem Ufipa-Plateau, SW Tanzania. - = Bayreuther Geowissenschaftliche Arbeiten 16: 186-205.

Vogel, C. (1998): Vulnerability and global environmental change. - LUCC Newsletter 3: 15-19.

ZedLITZ, H.A. von (1994): Traditional and Informal Social Security Systems and their Development Potential. - In: Dittrich, S. \& J. Petersen-Thumser (Hrsg.): Social Security in Africa - Proceedings of the International Conference held in Berlin, November 1993. Berlin-Tegel: ZWS Zentralstelle für Wirtschafts- und Sozialentwicklung: 233-261.

\section{Zusammenfassung: Gesellschaftliche Umverteilung, ein moralisches Muss? Verwundbarkeit und soziale Sicherung im Zeichen gesellschaftlichen Umbruchs - Beobachtungen aus Tansania in akteursorientierter Interpretation}

Der Aspekt gesellschaftlicher Umverteilung ist als ein Faktor, der für eine Verwundbarkeit durch Nahrungsdefizite bedeutsam ist, mit in den Vordergrund der Erforschung von Nahrungskrisen gerückt. Der Artikel wendet sich ihm zu und analysiert die Dynamik des diesbezüglichen Wandels in Tansania, die sich als Reaktion der Menschen auf eine Konfrontation mit rasch wechselnden und teilweise widersprüchlichen strukturellen Rahmenbedingungen darstellt. Die Perspektive der Analyse ist die von Akteuren in ihrem handelnd-gestaltenden Umgang mit den ihnen vorgegebenen Strukturen. Es wird die Frage beantwortet, in welcher Weise und für wen das Handeln mit den ihm zugrunde liegenden Strukturationsprozessen zu mehr oder weniger sozialer Sicherheit beiträgt.

Summary: Social Re-Allocation, a Moral Obligation? Vulnerability and social security under the impact of social change - an actor-oriented approach based on observations made in Tanzania

The aspect of social re-allocation as one of the factors determining vulnerability in food security has taken on a pole position in food-crisis research. The article focuses on this aspect and analyses the dynamics of change in social re-allocation in Tanzania, change which reflects the reactions of people who find themselves confronted with a rapidly changing and partly contradictory structural framework. The perspective of the analysis is that of actors who create and recreate the structural framework of their activity spheres. The question answered is in which way and for whom the actors and the underlying process of structuration contribute towards an improvement or deterioration of social security.

Résumé : La redistribution sociale: un devoir moral? Vulnérabilité et sécurité sociale dans un contexte de bouleversement social. Quelques observations sur la Tanzanie selon une interprétation centrée sur les acteurs

La redistribution sociale, en tant que facteur important de la vulnérabilité liée aux déficits alimentaires, est aussi un aspect mis en avant dans la recherche sur les crises alimentaires. L'article y est consacré et analyse la dynamique du changement observée à ce sujet en Tanzanie. Celle-ci se présente comme une réaction des hommes à une confrontation à des conditions structurelles en partie contradictoires et soumises à un changement rapide. La perspective retenue pour l'analyse est celle des acteurs considérés à travers leurs comportements face aux structures auxquelles ils sont confrontés. Il s'agit de répondre à la question suivante: comment et pour qui la manière d'agir sur les processus de structuration, sur lesquels elle est fondée, contribue à plus ou moins de sécurité sociale?

Prof. Dr. Sabine Tröger, Universität Bayreuth, Geographisches Institut, Universitätsstraße 30, D-95447 Bayreuth.

e-mail: Sabine.Troeger@uni-bayreuth.de

\section{Manuskripteingang/received/manuscrit entré le 2.10.2001 \\ Annahme zum Druck/accepted for publication/accepté pour l'impression: 25.2.2002}

